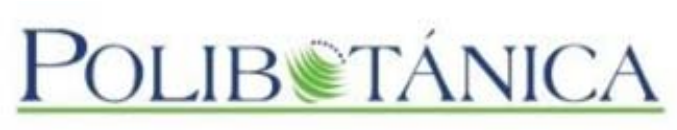

\title{
Polibotánica
}

ISSN electrónico: 2395-9525

polibotanica@gmail.com

Instituto Politécnico Nacional

México

http://www.polibotanica.mx

\section{EVALUACIÓN in vitro DE PRODUCTOS QUÍMICOS NO CONVENCIONALES PARA EL CONTROL DE Penicillium citrinum.}

\section{In vitro EVALUATION OF NON- CONVENTIONAL CHEMICALS FOR Penicillium citrinum CONTROL.}

González-Estrada, R.R., J. Vega-Arreguín, B.A. Robles-Villanueva, R.M. VelázquezEstrada, A. Ramos-Guerrero y P. Gutiérrez-Martínez.

EVALUACIÓN in vitro DE PRODUCTOS QUÍMICOS NO CONVENCIONALES PARA EL CONTROL DE Penicillium citrinum.

In vitro EVALUATION OF NON-CONVENTIONAL CHEMICALS FOR Penicillium citrinum CONTROL.

\section{POLIB ETANICA}

Instituto Politécnico Nacional
Núm. 49: 161-172 México. Enero 2020

DOI: $10.18387 /$ polibotanica.49.11 


\section{EVALUACIÓN in vitro DE PRODUCTOS QUÍMICOS NO CONVENCIONALES PARA EL CONTROL DE Penicillium citrinum.}

\section{In vitro EVALUATION OF NON-CONVENTIONAL CHEMICALS FOR Penicillium citrinum CONTROL.}

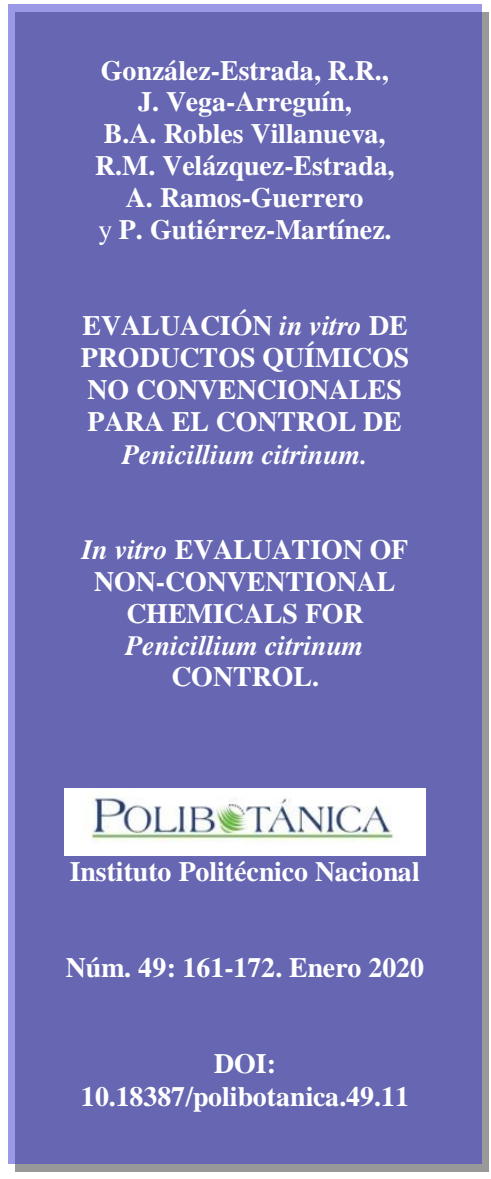

González-Estrada, R.R.

J. Vega-Arreguín,

B.A. Robles Villanueva,

R.M. Velázquez-Estrada,

A. Ramos-Guerrero

y P. Gutiérrez-Martínez.

EVALUACIÓN in vitro DE

PRODUCTOS QUÍMICOS

NO CONVENCIONALES

PA EL CONTROL DE

Penicillium citrinum.

In vitro EVALUATION OF

NON-CONVENTIONAL

CHEMICALS FOR

enicillium citrinum

CONTROL.

\section{POLIBETÁNICA}

Instituto Politécnico Naciona

Núm. 49: 161-172. Enero 2020

0.18387/polibotanica.49.11

\author{
R.R. González-Estrada \\ Tecnológico Nacional de México/I. T. Tepic \\ Av. Tecnológico \# 2595, Col. Lagos del Country. \\ CP 63175 Tepic, Nayarit. México. \\ J. Vega-Arreguín \\ Universidad Nacional Autónoma de México \\ Escuela Nacional de Estudios Superiores Unidad León \\ Blvd. UNAM 2011, Col. Predio el Saucillo y El Potrero, \\ Comunidad de Los Tepetates, León 37684, Guanajuato, México.
}

B.A. Robles Villanueva

R.M. Velázquez-Estrada

A. Ramos-Guerrero

P. Gutiérrez-Martínez / pgutierrez@ittepic.edu.mx Tecnológico Nacional de México/I. T. Tepic Av. Tecnológico \# 2595, Col. Lagos del Country. CP 63175

Tepic, Nayarit. México.

RESUMEN: El ajo (Allium sativum L.) es un cultivo que esta expuesto al ataque de patógenos del género Penicillium provocando pérdidas económicas importantes. En este estudio, $P$. citrinum fue aislado a partir de tejidos de ajo e identificado mediante herramientas moleculares. Se evaluó la capacidad antifúngica de tratamientos de peróxido de hidrógeno, SP, BS y quitosano aplicado de forma individual (crecimiento micelial y germinación) a diferentes concentraciones, así como sus combinaciones (tomando en cuenta los resultados de la aplicación individual) en el control in vitro de $P$. citrinum en términos de crecimiento micelial, esporulación y germinación. La aplicación de, $\mathrm{H}_{2} \mathrm{O}_{2}$ al $0.5 \%$, el BS al $3 \%$ y el quitosano al $1 \%$ resultaron efectivos en la inhibición (100\%) del desarrollo del patógeno. Se observó un efecto sinérgico de los tratamientos evaluados en combinación a baja concentración de quitosano $(0.05 \%)$. Por lo tanto, los tratamientos evaluados pueden ser una alternativa viable y amigable con el medio ambiente el control de $P$. citrinum en ajo.

Palabras clave: antifúngico, sistemas alternativos, ajo.

ABSTRACT: Garlic (Allium sativum L.), is a crop that is exposed to the attack by important pathogens of the genus Penicillium causing significant economic losses. In this study, $P$. citrinum was isolated and identified by molecular tools. The antifungal capacity of hydrogen peroxide, SP, BS and chitosan treatments applied individually (mycelial growth and germination) at different concentrations as well as their combinations (taking into account the results of the individual application) for controlling in vitro of $P$. citrinum in terms of mycelial growth, sporulation and germination. The application of $0.5 \% \mathrm{H}_{2} \mathrm{O}_{2}, 3 \% \mathrm{SB}$ and $1 \%$ chitosan were effective in inhibiting the development of the pathogen $(100 \%)$. Synergistic effects of the treatments were observed when they were applied in combination with chitosan at low 
concentration $(0.05 \%)$. Thus, the treatments evaluated in this study can be a viable and ecofriendly alternative for controlling $P$. citrinum in garlic.

Key words: antifungal, alternative systems, garlic.

\title{
INTRODUCCIÓN
}

El ajo (Allium sativum L.) es considerado uno de los cultivos más rentables en México (MacíasDuarte et al., 2010). Debido al tamaño del bulbo y su larga vida de anaquel, se crean dificultades en el manejo de enfermedades de postcosecha causadas por fitopatógenos ocasionando pérdidas económicas para los agricultores (Patón et al., 2017). Diferentes especies de Penicillium han sido reportadas como patógenos de ajo en diferentes países incluyendo a México (Cavagnaro et al., 2005; Fuentes et al., 2013; Overy et al., 2005). En la actualidad, el uso de fungicidas sintéticos es la estrategia principal para el control de patógenos durante la poscosecha (Singh \& Sharma, 2018). Debido a lo anterior es necesaria la búsqueda de fungicidas que no generen efectos perjudiciales al medio ambiente y que sean efectivos contra fitopatógenos. En este sentido, el quitosano es el segundo polisacárido más abundante en la naturaleza, posee actividad antimicrobiana, es biodegradable y no es tóxico para los humanos ni animales (Gutierrez-Martinez et al., 2018). El quitosano ha sido efectivo contra patógenos aislados de guanábana (Ramos-Guerrero et al., 2018), aguacate (Chávez-Magdaleno et al., 2018), papaya (Hernández-López et al., 2018), entre otros. Por su parte, trabajos previos han demostrado un fuerte efecto inhibitorio al aplicar el peróxido de hidrógeno como estrategia para el control de patógenos como Listeria monocytogenes y Salmonella typhimurium en melón y arándanos respetivamente (Li et al., 2013; Upadhyay et al., 2013). También, es ampliamente conocida la actividad antifúngica de algunas sales orgánicas como son el BS (Bicarbonato de sodio) y el SP (Sorbato de potasio) en la inhibición de la germinación de esporas, la elongación del tubo germinal y la producción de enzimas pectinolíticas en varios patógenos tanto en pruebas in vitro como en evaluaciones in vivo (Mejía-Teniente et al., 2013; Yao et al., 2004; Youssef et al., 2012; Youssef \& Roberto, 2014). Por lo anterior, se planteó como objetivo de este proyecto estudiar el efecto de Q (Quitosano), $\mathrm{H}_{2} \mathrm{O}_{2}$ y dos sales orgánicas aplicados de forma individual y en combinación como productos químicos alternativos en el control in vitro de $P$. citrinum.

\section{MATERIAl y MÉTOdos}

\begin{abstract}
Aislamiento del patógeno
Las muestras de ajo morado (Allium sativum L.) fueron recolectadas en parcelas de la ciudad de León, Guanajuato, sin presencia de enfermedades ni daño mecánico. Los bulbos se colocaron en cámara húmeda (HR 90-95\%) a $25^{\circ} \mathrm{C}$ hasta el desarrollo de la enfermedad. Se realizaron pequeños cortes en las muestras de tejido con $50 \%$ de superficie sana y $50 \%$ de superficie dañada con la finalidad de garantizar que se tiene tejido infectado. Posteriormente, las secciones de tejido se desinfectaron superficialmente en una solución de hipoclorito de sodio al $1 \%$ por 3 minutos, se enjuagaron en agua destilada estéril y se colocaron sobre papel filtro. Las secciones de tejido fueron sembradas en cajas de Petri con medio APD (Agar papa dextrosa) (Difco ${ }^{\circledR}$ ) e incubadas a $25^{\circ} \mathrm{C} \pm 2$ por siete días. Se realizaron explantes en función de los tipos y coloraciones de micelio desarrollados, hasta obtener un cultivo puro (Ochoa Fuentes et al., 2012).

\section{Identificación morfológica de Penicillium sp.}

Se recolectaron porciones de micelio con esporas que fueron observados al microscopio óptico (Motic, BA300) empleando los objetivos 40 y 100X, la identificación morfológica se realizó con base en las claves taxonómicas de Barnett \& Hunter (1998) y Visagie et al. (2014).
\end{abstract}




\section{Identificación molecular de Penicillium sp.}

La extracción de ADN se realizó mediante el método propuesto por Doyle \& Doyle (1990). Posteriormente, el ADN se amplificó mediante el método de reacción en cadena de la polimerasa (PCR, por sus siglas en inglés), empleando las regiones internas transcritas ITS15.8S-ITS2 utilizando el par de iniciadores de secuencia ITS4/ ITS5 siguiendo el protocolo propuesto por White et al. (1990). Las condiciones de la reacción de PCR fueron: 1 ciclo de desnaturalización inicial a $94^{\circ} \mathrm{C}$ por $5 \mathrm{~min}, 30$ ciclos a $95^{\circ} \mathrm{C}$ por 10 segundos; $57^{\circ} \mathrm{C}$ por 30 segundos; $72^{\circ} \mathrm{C}$ por $2 \mathrm{~min}$ y 1 ciclo de extensión final a $72^{\circ} \mathrm{C}$ por $5 \mathrm{~min}$. Los productos amplificados se visualizaron en un gel de agarosa al $1 \%$ mediante electroforesis. El producto de PCR fue purificado empleando PureLink ${ }^{\circledR}$ (Quick Gel Extraction and PCR Purification Combo Kit). Los pares de base obtenidos se compararon con las secuencias reportadas en la base de datos del banco de genes de NCBI (National Center for Biotechnology Information) mediante el programa BLAST.

\section{Preparación de tratamientos}

Las soluciones de quitosano peso molecular medio (desacetilación al 75-85\%) (Sigma-Aldrich, St. Louis, MO, EE. UU.) se prepararon a 0.1, 0.5, 1.0 y $1.5 \%(\mathrm{p} / \mathrm{v})$ en $100 \mathrm{~mL}$ de ácido acético al 2\% (v/v), se agitó por 24 horas a temperatura ambiente, las soluciones se ajustaron a pH de 5.5 agregando $\mathrm{NaOH}$ al $1 \mathrm{~N}$ y se añadieron $100 \mu \mathrm{L}$ de Tween 80 (Ramos-Guerrero et al., 2018). Las soluciones de $\mathrm{H}_{2} \mathrm{O}_{2}$ (Sigma-Aldrich, St. Louis, MO, EE. UU.) se prepararon al 0.1, 0.5, 1.0, y $1.5 \%(\mathrm{v} / \mathrm{v})$, por su parte las soluciones de BS (Jalmek, México) y SP (Jalmek, México) se prepararon a las siguientes concentraciones: $1.0,1.5,2.0,2.5$ y $3.0 \%(\mathrm{p} / \mathrm{v})$, diluyendo el reactivo comercial en agua destilada. Todas las soluciones fueron esterilizadas en autoclave. Una vez determinada la efectividad antifúngica de los tratamientos aplicados de forma individual se procedió a seleccionar las combinaciones de tratamientos considerando que las concentraciones deberian estar por debajo del $50 \%$ (de las más efectivas) quedando de la siguiente manera: $\quad 0.05 \% \mathrm{Q}+0.25 \% \mathrm{H}_{2} \mathrm{O}_{2}, \quad 0.05 \% \mathrm{Q}+1.0 \% \mathrm{SP}, \quad 0.05 \% \mathrm{Q}+2.0 \% \mathrm{BS}$, $0.05 \% \mathrm{Q}+2.0 \% \mathrm{BS}+1.0 \% \mathrm{SP}+0.25 \% \mathrm{H}_{2} \mathrm{O}_{2}, \quad 1.0 \% \mathrm{SP}+0.25 \% \mathrm{H}_{2} \mathrm{O}_{2}, \quad 2.0 \% \mathrm{BS}+1.0 \% \mathrm{SP} \quad$ у $0.25 \% \mathrm{H}_{2} \mathrm{O}_{2}+2.0 \% \mathrm{BS}$.

\section{Evaluación de efectividad in vitro}

Para evaluar el crecimiento micelial se tomó un disco de $7 \mathrm{~mm}$ de diámetro proveniente de la periferia de la colonia del hongo en estudio con crecimiento activo. Posteriormente, el disco se colocó en el centro de una caja de Petri con medio APD adicionado con el tratamiento; el control consistió en cajas APD-sin tratamiento. Una vez inoculadas las cajas Petri se incubaron a $25^{\circ} \mathrm{C}$ y se midió el diámetro micelial en crecimiento con un vernier digital (Truper ${ }^{\mathrm{MR}}$ ) cada 24 h, la prueba se detuvo cuando el hongo en la caja Petri control cubrió el $100 \%$ de la superficie. Los resultados se expresaron en porcentaje de inhibición del crecimiento micelial empleando la fórmula propuesta por Yahyazadeh et al. (2008). La prueba de esporulación se llevó a cabo a las $120 \mathrm{~h}$ de incubación del hongo fitopatógeno, empleando las mismas cajas de APD-con tratamientos. Se adicionaron $10 \mathrm{ml}$ de agua destilada estéril y con una varilla de vidrio se raspo la superficie de las cajas; las esporas colectadas fueron transferidas a un tubo de ensayo. La suspensión de esporas se agitó en Vortex, se tomaron $20 \mu \mathrm{l}$ y se colocaron sobre una cámara de Neubauer para cuantificar las esporas en un microscopio óptico (40X). Para evaluar la germinación se preparó una suspensión de esporas de una colonia del hongo de 8 días; ajustando la concentración a $1 \times 10^{8}$ esporas $/ \mathrm{mL}$. Se prepararon tubos Eppendorf que contenían medio APD líquido con los tratamientos. Cada tubo eppendorf fue inoculado con la misma concentración de esporas, posteriormente se incubaron a $26^{\circ} \mathrm{C}$ en agitación constante durante 14h. Se contaron las esporas germinadas y las no germinadas en una cámara de Neubauer cada dos horas hasta que el control germinó al 100\%. Una espora ha germinado cuando el tubo germinativo sea igual o mayor a la longitud de ésta (Yao et al., 2004). Los resultados se reportaron en número de esporas $\mathrm{ml}^{-1}$. Todos los ensayos se realizaron por triplicado y los experimentos se repitieron dos veces. 


\section{Análisis estadístico}

Para evaluar el crecimiento micelial y germinación de los tratamientos aplicados de forma individual se aplicó un diseño unifactorial de bloques. Se consideraron los diferentes factores que conforman el sistema de control: quitosano (con 5 niveles: $0.05 \%, 0.1 \%, 0.5 \%, 1.0 \%$ y $1.5 \%$ ), peróxido de hidrógeno (con 7 niveles: $0.25 \%, 0.5 \%, 1.0 \%, 1.5 \%, 2.0 \%, 2.5 \%$ y $3.0 \%$ ), bicarbonato de sodio (con 5 niveles: $1.0 \%, 1.5 \%, 2.0 \%, 2.5 \%$ y $3.0 \%$ ), sorbato de potasio (con 5 niveles: $1.0 \%, 1.5 \%, 2.0 \%, 2.5 \%$ y $3.0 \%)$. Para las combinaciones se aplicó un diseño unifactorial de bloques para evaluar el crecimiento micelial, la esporulación y germinación, con el factor tratamiento con 7 niveles $\left(0.05 \% \mathrm{Q}+0.25 \% \mathrm{H}_{2} \mathrm{O}_{2}, 0.05 \% \mathrm{Q}+1.0 \% \mathrm{SP}, 0.05 \% \mathrm{Q}+2.0 \% \mathrm{BS}\right.$, $0.05 \% \mathrm{Q}+2.0 \% \mathrm{BS}+1.0 \% \mathrm{SP}+0.25 \% \mathrm{H}_{2} \mathrm{O}_{2}, \quad 1.0 \% \mathrm{SP}+0.25 \% \mathrm{H}_{2} \mathrm{O}_{2}, \quad 2.0 \% \mathrm{BS}+1.0 \% \mathrm{SP} \quad$ y $\left.0.25 \% \mathrm{H}_{2} \mathrm{O}_{2}+2.0 \% \mathrm{BS}\right)$. Los resultados obtenidos se analizaron mediante un análisis de varianza (ANOVA, por sus siglas en inglés) donde de se realizaron comparaciones de medias por prueba de Tukey $(\alpha<0.05)$ empleando el paquete estadístico GraphPad.

\section{REsultados}

\section{Identificación morfológica y molecular del patógeno}

La colonia del hongo se desarrolló sobre medio APD a $25^{\circ} \mathrm{C}$ de 5 a 9 días. El color inicialmente es blanco virando a verde-azulado. La textura de las colonias es pulverulenta y plegada (fig. 1A). El reverso de la colonia es amarillento (fig. 1B). A nivel microscópico el hongo presenta conidióforos simples, penicilados, que termina en un grupo de fiálides ampuliformes y conidias. Presenta ramificaciones secundarias conocidas como métulas que se forman sobre los conidios; de tres a cinco métulas por estípite (fig. 1C). Las conidias o esporas son redondas, unicelulares y se observan como cadenas no ramificadas en el extremo de las fiálides. El patrón de ramificación observado fue biverticilada (Pitt \& Hocking, 2009; Visagie et al., 2014).

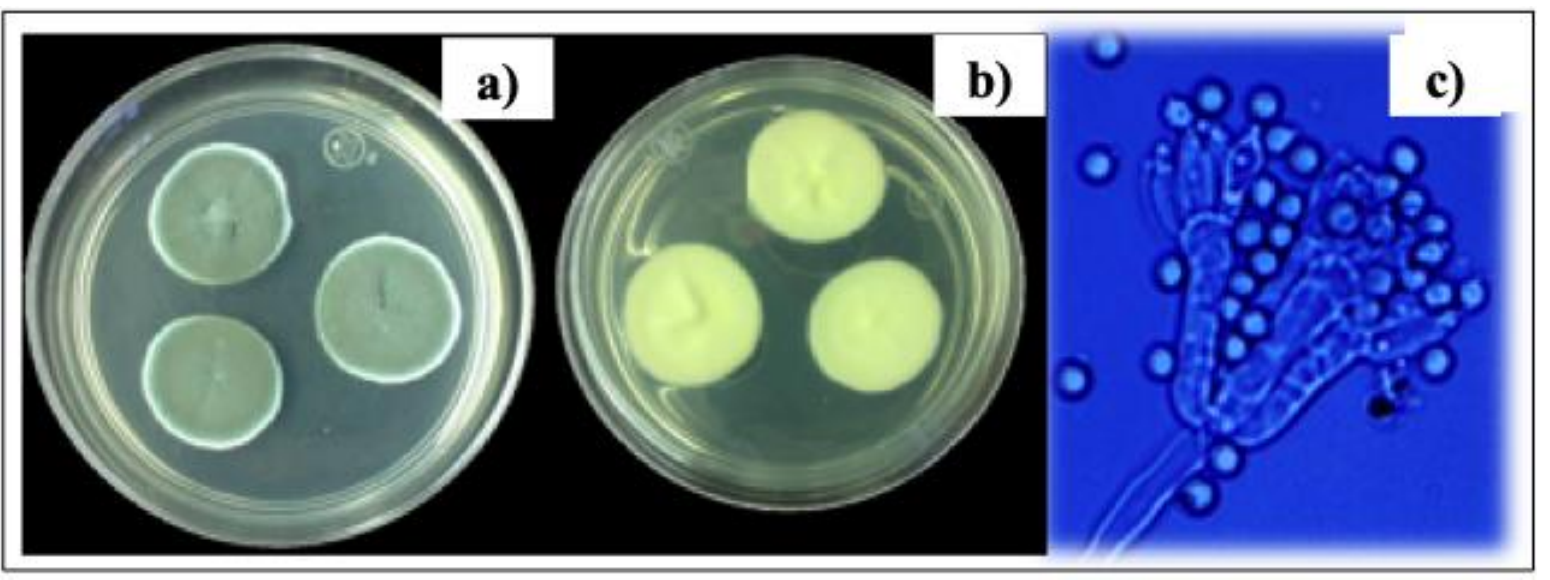

Fig 1. Morfología de Penicillium sp. Estructura macroscópica: a) Anverso, b) Reverso y c) Estructura microscópica

La amplificación de ADN, por medio de PCR, produjo un producto de aproximadamente 701 pares de bases lo que permitió la identificación de la especie del hongo en estudio. Los porcentajes de similitud obtenidos en la base de datos del GenBank fueron del 99.39\% (número de accesión: HQ891112.1) para Penicillium citrinum LXL-24, especie no reportada como patógeno de ajo. 


\section{Evaluación de efectividad in vitro}

La aplicación de $\mathrm{H}_{2} \mathrm{O}_{2}$ inhibió en su totalidad el desarrollo del patógeno empleando concentraciones desde 0.5 hasta el $3.0 \%$ (fig. 2a), observándose una diferencia significativa (p $<0.05$ ) entre las concentraciones evaluadas. Con respecto al BS, los resultados muestran diferencia significativa $(\mathrm{p}<0.05)$ entre las concentraciones aplicadas (fig. $2 b)$ y al aplicar la mayor concentración analizada (3\%) se observó una total inhibición del hongo.

Por otra parte, los resultados muestran diferencia significativa $(\mathrm{p}<0.05)$ entre las concentraciones aplicadas de SP (Fig. 2c). La máxima inhibición (87\%) se obtuvo con una concentración del 3\%.

Stanojevic et al. (2009) reportaron resultados similares al aplicar SP al 1.5\% en el control de $P$. italicum.

En los tratamientos con quitosano se observa que al utilizar la concentración del $1 \%$ se logra una inhibición del $100 \%$ en el crecimiento radial del patógeno (fig. 2d). El análisis estadístico de los datos muestra diferencia significativa entre los tratamientos $(\mathrm{p}<0.05)$.

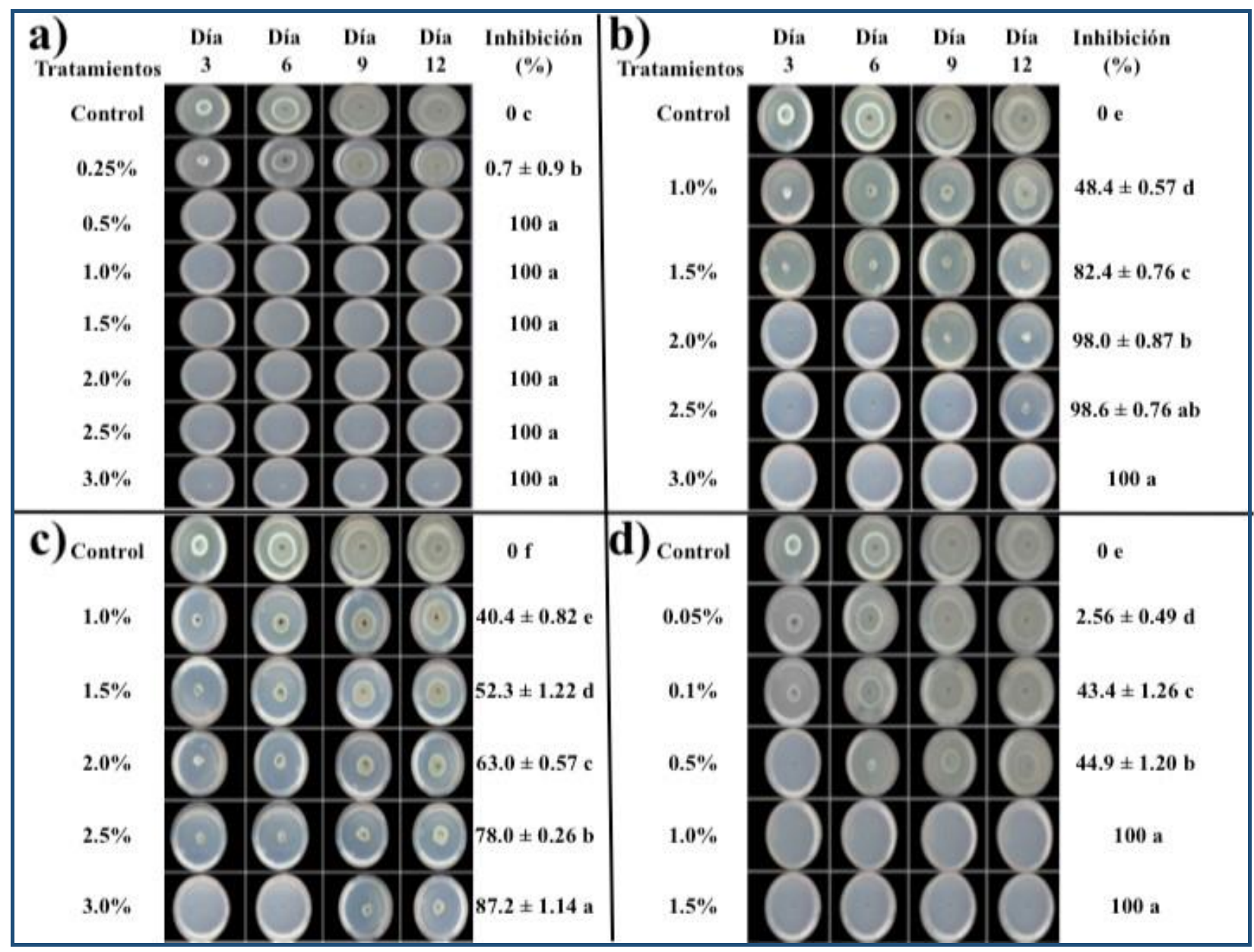

Fig 2. Efecto de los diferentes tratamientos en el desarrollo micelial de $P$. citrinum en APD. a) $\mathrm{H}_{2} \mathrm{O}_{2}$, B) BS, c) SP y d) Quitosano. 
En las combinaciones de tratamientos, el análisis estadístico de los datos muestra diferencias significativas entre los tratamientos $(\mathrm{p}<0.05)$. Se obtuvieron resultados favorables para las diferentes combinaciones probadas en la inhibición in vitro de P. citrinum con la excepción del tratamiento quitosano y peróxido de hidrógeno en la que la concentración de ambos compuestos no fue suficiente para presentar un control sobre el patógeno (fig. 3).

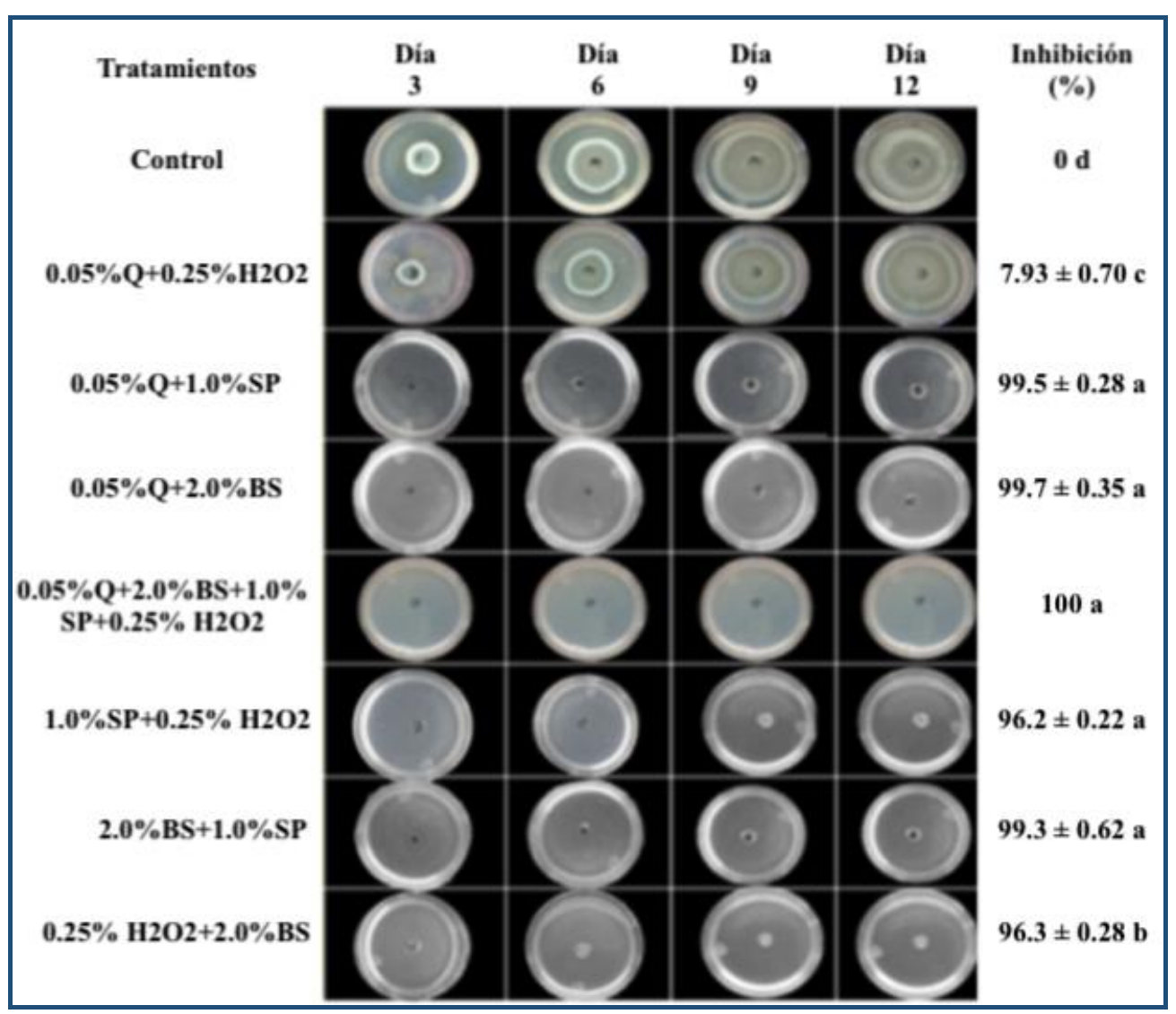

Fig 3. Efecto de la combinación de los diferentes tratamientos en el desarrollo micelial de P. citrinum

La germinación fue totalmente inhibida mediante la aplicación de los tratamientos individuales, excepto con la aplicación de quitosano $(0.05 \%$ y $0.1 \%)$ (tabla 1$)$. La combinación de tratamientos fue efectiva en la inhibición de la producción del tubo germinal (tabla 2).

La combinación de tratamientos resultó efectiva para el control de la esporulación, observándose diferencia estadística significativa, solo el tratamiento de quitosano combinado con $\mathrm{H}_{2} \mathrm{O}_{2}$ no resulto efectivo para detener el proceso de esporulación al evidenciar incluso una mayor esporulación con respecto al control (tabla 2). 
Tabla 1. Efecto de los tratamientos individuales en la germinación de conidios de P. citrinum.

\begin{tabular}{|c|c|}
\hline & Germinación de esporas* $(\%)$ \\
\hline Control & $100 \mathrm{a}$ \\
\hline $0.25 \% \mathrm{H}_{2} \mathrm{O}_{2}$ & $0.0 \pm 0.0 \mathrm{~b}$ \\
\hline $0.50 \% \mathrm{H}_{2} \mathrm{O}_{2}$ & $0.0 \pm 0.0 \mathrm{~b}$ \\
\hline $1.0 \% \mathrm{H}_{2} \mathrm{O}_{2}$ & $0.0 \pm 0.0 \mathrm{~b}$ \\
\hline $1.5 \% \mathrm{H}_{2} \mathrm{O}_{2}$ & $0.0 \pm 0.0 \mathrm{~b}$ \\
\hline $2.0 \% \mathrm{H}_{2} \mathrm{O}_{2}$ & $0.0 \pm 0.0 \mathrm{~b}$ \\
\hline $3.0 \% \mathrm{H}_{2} \mathrm{O}_{2}$ & $0.0 \pm 0.0 \mathrm{~b}$ \\
\hline Control & $100 \mathrm{a}$ \\
\hline $1.0 \% \mathrm{BS}$ & $0.0 \pm 0.0 \mathrm{~b}$ \\
\hline $1.5 \% \mathrm{BS}$ & $0.0 \pm 0.0 \mathrm{~b}$ \\
\hline $2.0 \% \mathrm{BS}$ & $0.0 \pm 0.0 \mathrm{~b}$ \\
\hline $2.5 \% \mathrm{BS}$ & $0.0 \pm 0.0 \mathrm{~b}$ \\
\hline $3.0 \% \mathrm{BS}$ & $0.0 \pm 0.0 \mathrm{~b}$ \\
\hline Control & $100 \mathrm{a}$ \\
\hline $1.0 \% \mathrm{SP}$ & $0.0 \pm 0.0 \mathrm{~b}$ \\
\hline $1.5 \% \mathrm{SP}$ & $0.0 \pm 0.0 \mathrm{~b}$ \\
\hline $2.0 \% \mathrm{SP}$ & $0.0 \pm 0.0 \mathrm{~b}$ \\
\hline $2.5 \% \mathrm{SP}$ & $0.0 \pm 0.0 \mathrm{~b}$ \\
\hline $3.0 \% \mathrm{SP}$ & $0.0 \pm 0.0 \mathrm{~b}$ \\
\hline Control & $100 \mathrm{a}$ \\
\hline $0.05 \% \mathrm{Q}$ & $85 \pm 5.0 \mathrm{ab}$ \\
\hline $0.1 \% \mathrm{Q}$ & $84 \pm 6.0 \mathrm{ab}$ \\
\hline $0.5 \% \mathrm{Q}$ & $0.0 \pm 0.0 \mathrm{~b}$ \\
\hline $1.0 \% \mathrm{Q}$ & $0.0 \pm 0.0 \mathrm{~b}$ \\
\hline $1.5 \% \mathrm{Q}$ & $0.0 \pm 0.0 \mathrm{~b}$ \\
\hline
\end{tabular}

* Letras diferentes en la columna indican diferencias significativas (Tukey, $\mathrm{p}<0.05$ )

Tabla 2. Efecto de los tratamientos combinados en la esporulación y germinación de conidios de P. citrinum.

\begin{tabular}{|c|c|c|}
\hline Tratamiento & $\begin{array}{c}\text { Esporulación* } \\
\text { esporas x10 } / \mathbf{m L})\end{array}$ & $\begin{array}{c}\text { Germinación de esporas* } \\
(\%)\end{array}$ \\
\hline Control & $4.36 \pm 1.32 \mathrm{~b}$ & $00 \mathrm{a}$ \\
\hline $0.05 \% \mathrm{Q}+0.25 \% \mathrm{H}_{2} \mathrm{O}_{2}$ & $0 \mathrm{~b}$ \\
\hline $0.05 \% \mathrm{Q}+1.0 \% \mathrm{SP}$ & $0.66 \pm 0.14 \mathrm{a}$ & $0 \mathrm{~b}$ \\
\hline $0.05 \% \mathrm{Q}+2.0 \% \mathrm{BS}$ & $0.0035 \pm 0.002 \mathrm{c}$ & $0 \mathrm{~b}$ \\
\hline $0.05 \% \mathrm{Q}+2.0 \% \mathrm{BS}+1.0 \% \mathrm{SP}+0.25 \% \mathrm{H}_{2} \mathrm{O}_{2}$ & $0 \mathrm{c}$ & $0 \mathrm{~b}$ \\
\hline $1.0 \% \mathrm{SP}+0.25 \% \mathrm{H}_{2} \mathrm{O}_{2}$ & $0.007 \pm 0.003 \mathrm{c}$ \\
\hline $2.0 \% \mathrm{BS}+1.0 \% \mathrm{SP}$ & $0.006 \pm 0.002 \mathrm{c}$ & $0 \mathrm{~b}$ \\
\hline $0.25 \% \mathrm{H}_{2} \mathrm{O}_{2}+2.0 \% \mathrm{BS}$ & $0.007 \pm 0.004 \mathrm{c}$ & $0 \mathrm{~b}$ \\
\hline
\end{tabular}

* Letras diferentes en la columna indican diferencias significativas (Tukey, $\mathrm{p}<0.05$ ) 


\section{DISCUSIÓN}

La efectividad del $\mathrm{H}_{2} \mathrm{O}_{2}$ puede ser atribuida a daño celular masivo a nivel macromolecular; que incluye la ruptura de las capas de la membrana, inhibición enzimática, oxidación de nucleósidos, la interrupción de la síntesis de proteínas y, en última instancia, la muerte celular (Finnegan et al., 2010). Estos resultados son superiores a lo reportado por Qin et al. (2011), ya que al aplicar $\mathrm{H}_{2} \mathrm{O}_{2}$ al $1 \%$, reportaron un $60 \%$ de inhibición del desarrollo micelial en $P$. expansum.

Se ha atribuido la capacidad inhibitoria del BS a una modificación del pH del entorno de crecimiento de los patógenos, lo que da lugar a la inactivación de enzimas extracelulares del fitopatógeno, un incremento en el estrés osmótico y una reducción en la presión de turgencia de la célula; resultado en un colapso y encogimiento de las hifas. En un reporte previo, la aplicación de BS al $0.6 \%$ inhibió el desarrollo micelial de P. expansum un 40\% (Lai et al., 2015).

El efecto inhibitorio del SP parece ser debido al decremento del $\mathrm{pH}$ intracelular y a la ionización de moléculas ácidas afectando de esta manera el desarrollo del hongo (Gregori et al., 2008; Stanojevic et al., 2009).

La eficacia por la cual el quitosano muestra un efecto inhibitorio sobre el crecimiento micelial del hongo podría ser explicado por varios mecanismos de acción; el primero de ellos es debido a la naturaleza policatiónica del quitosano, que interacciona con los fosfolípidos que se encuentran en la pared celular del patógeno, lo que lleva a un aumento en la permeabilidad de la membrana plasmática y en segunda instancia se cree que el quitosano puede entrar en la célula fúngica, interactuar con el ADN, y alterar su conformación, por tanto, inhibir la síntesis de mRNA y proteínas (Kong et al., 2010; Ngo et al., 2015). Estos resultados concuerdan con lo informado por Wang et al. (2014) en el que se probaron concentraciones de $0.1 \%(1 \mathrm{mg} / \mathrm{ml})$, $0.5 \%(5 \mathrm{mg} / \mathrm{ml})$ y $1 \%(10 \mathrm{mg} / \mathrm{ml})$ de quitosano sobre el crecimiento micelial de $P$. expansum patógeno del fruto de azufaifo (Ziziphus jujuba); reportando que la efectividad era dependiente de la concentración de quitosano empleada.

Respecto a las combinaciones de tratamientos, los resultados sugieren una interacción negativa entre el quitosano y el peróxido de hidrógeno, en este sentido Qin et al. (2002) mencionan que el $\mathrm{H}_{2} \mathrm{O}_{2}$ puede afectar en el quitosano el grado de polimerización y cambios en la estructura química del biopolímero como la formación de grupos carboxilo los cuales están relacionados con un proceso de desaminación debido a reacciones con radicales.

El mecanismo de acción de acción del quitosano para inhibir el proceso de germinación esta relacionado con las interacciones entre las cargas de los grupos $-\mathrm{NH}_{3}$ y las cargas negativas en la membrana celular causando una modificación de la permeabilidad de la misma, retardando el proceso de germinación de la espora (Li et al., 2015; Wang et al., 2014). Por su parte, $\mathrm{El}_{2} \mathrm{O}_{2}$ es efectivo debido a que los radicales libres oxidan enzimas y proteínas provocando ruptura de cadenas de ADN (Minibayeva, Beckett, \& Kranner, 2015). Se ha reportado que el BS aumenta el estrés osmótico de la célula provocando cambios estructurales como colapso y contracción de esporas (Lai et al., 2015). La actividad antifúngica del SP esta relacionado con la afectación del gradiente electroquímico de la membrana citoplasmática (transporte activo de sustancias), lo que a su vez inhibe el transporte de aminoácidos y de procesos enzimáticos (Youssef et al., 2012). Estos resultados son prometedores debido a que cualquier agente que pueda controlar el proceso de germinación coadyuva a evitar la diseminación del patógeno y disminuir la posibilidad de infecciones.

Finalmente, Hernández et al. (2007) y Reddy et al. (1998) mencionan que la excesiva esporulación del hongo sugiere una concentración sub-letal del tratamiento lo que da lugar a un proceso de estrés provocando la activación de mecanismos de defensa del patógeno asegurando 
la supervivencia hasta encontrar las condiciones favorables para su desarrollo. La eficacia de la combinación de los tratamientos puede ser atribuida en la capacidad de los compuestos de afectar el desarrollo del micelio y en consecuencia la formación estructuras de dispersión.

\section{CONCLUSIONES}

De acuerdo a los datos y análisis se concluye que $P$. citrium es el agente causal de la pudrición azul en ajo morado (Allium sativum $\mathrm{L}$ ). Además, el $\mathrm{H}_{2} \mathrm{O}_{2}$ al $0.5 \%$, el $\mathrm{BS}$ al $3 \%$ así como el quitosano al $1 \%$ resultaron efectivos en la inhibición del crecimiento micelial y germinación del patógeno al $100 \%$. Por su parte, en las combinaciones evaluadas se observó un efecto sinérgico empleando las concentraciones mínimas de cada compuesto a excepción de quitosano $-\mathrm{H}_{2} \mathrm{O}_{2}$. Debido a lo anterior, los tratamientos evaluados pueden ser una alternativa ecológica efectiva para el control de $P$. citrinum en ajo morado.

\section{AGRADECIMIENTOS}

Al Consejo Nacional de Ciencia y Tecnología (CONACYT) por la beca para estudios de maestría.

\section{LITERATURA CITADA}

Barnett, H. L., \& Hunter, B. B. (1998). Illustrated genera of imperfect fungi. American Phytopathological Society (APS Press). https://doi.org/https://doi.org/10.1007/s00299007-0368-x

Cavagnaro, P. F., Camargo, A., Piccolo, R. J., Lampasona, S. G., Burba, J. L., \& Masuelli, R. W. (2005). Resistance to Penicillium hirsutum Dierckx in garlic accessions. European Journal of Plant Pathology, 112(2), 195-199.

Chávez-Magdaleno, Mireya Esbeiddy, G.-E. R. R., Maribel, A. R., \& Gutie, P. P. (2018). Effect of pepper tree (Schinus molle) essential oil-loaded chitosan bio-nanocomposites on postharvest control of Colletotrichum gloeosporioides and quality evaluations in avocado (Persea americana) cv . Hass. https://doi.org/10.1007/s10068-018-0410-5

Doyle, J. J., \& Doyle, J. L. (1990). Isolation ofplant DNA from fresh tissue. Focus, 12(13), 39-40.

Finnegan, M., Linley, E., Denyer, S. P., McDonnell, G., Simons, C., \& Maillard, J.-Y. (2010). Mode of action of hydrogen peroxide and other oxidizing agents: differences between liquid and gas forms. Journal of Antimicrobial Chemotherapy, 65(10), 2108-2115.

Fuentes, Y. M. O., Ortiz, J. C. D., Chávez, E. C., Castillo, F. D. H., Olivas, A. F., Morales, G. G., ... Guerra, R. R. (2013). The first report of Fusarium proliferatum causing garlic bulb rots in Mexico. African Journal of Agricultural Research, 8(6), 570-573.

Gregori, R., Borsetti, F., Neri, F., Mari, M., \& Bertolini, P. (2008). Effects of potassium sorbate on postharvest brown rot of stone fruit. Journal of Food Protection, 71(8), 1626-1631.

Gutierrez-Martinez, P., Ledezma-Morales, A., Del, L., Romero-Islas, C., Ramos-Guerrero, A., Romero-Islas, J., ... González-Estrada, R. (2018). Antifungal Activity of Chitosan against Postharvest Fungi of Tropical and Subtropical Fruits. https://doi.org/10.5772/intechopen.76095

Hernández-Lauzardo, A. N., Hernández-Martínez, M., Velázquez-del Valle, M.G., GuerraSánchez, M.G. y Melo-Giorgana, G. E. (2007). Actividad Antifúngica del Quitosano en el Control de Rhizopus stolonifer ( Ehrenb .: Fr .) Vuill. y Mucor spp. Revista Mexicana de Fitopatología, 25(September), 109-113.

Hernández-López, M., Guillén-Sánchez, J., Bautista-Baños, S., \& Guillén-Sánchez, D. (2018). Evaluation of biodegradable films for postharvest control of fungi in papaya. Cultivos Tropicales, 39(1), 52-60. 
Kong, M., Chen, X. G., Xing, K., \& Park, H. J. (2010). Antimicrobial properties of chitosan and mode of action: a state of the art review. International Journal of Food Microbiology, 144(1), 51-63. https://doi.org/https://doi.org/10.1016/j.ijfoodmicro.2010.09.012

Lai, T., Bai, X., Wang, Y., Zhou, J., Shi, N., \& Zhou, T. (2015). Inhibitory effect of exogenous sodium bicarbonate on development and pathogenicity of postharvest disease Penicillium expansum. Scientia Horticulturae, 187, 108-114.

Li, H., Wang, Y., Liu, F., Yang, Y., Wu, Z., Cai, H., ... Li, P. (2015). Effects of chitosan on control of postharvest blue mold decay of apple fruit and the possible mechanisms involved. Scientia Horticulturae, 186, 77-83.

Li, Y., \& Wu, C. (2013). Enhanced inactivation of Salmonella typhimurium from blueberries by combinations of sodium dodecyl sulfate with organic acids or hydrogen peroxide. Food Research International, 54(2), 1553-1559.

Macías-Duarte, R., Grijalva-Contreras, R. L., \& Robles-Contreras, F. (2010). Productividad y calidad de variedades de ajo (Allium sativium L.) bajo condiciones desérticas en Caborca, Sonora. Biotecnia, 12(1), 44-54.

Mejía-Teniente, L., Durán-Flores, F. de D., Chapa-Oliver, A., Torres-Pacheco, I., CruzHernández, A., González-Chavira, M., ... Guevara-González, R. (2013). Oxidative and molecular responses in Capsicum annuum L. after hydrogen peroxide, salicylic acid and chitosan foliar applications. International Journal of Molecular Sciences, 14(5), 10178-10196.

Minibayeva, F., Beckett, R. P., \& Kranner, I. (2015). Roles of apoplastic peroxidases in plant response to wounding. Phytochemistry, 112, 122-129.

Ngo, D.-H., Vo, T.-S., Ngo, D.-N., Kang, K.-H., Je, J.-Y., Pham, H. N.-D., ... Kim, S.-K. (2015). Biological effects of chitosan and its derivatives. Food Hydrocolloids, 51, 200-216. https://doi.org/10.1016/j.foodhyd.2015.05.023

Ochoa Fuentes, Y. M., Cerna Chávez, E., Gallegos Morales, G., Landeros Flores, J., Delgado Ortiz, J. C., Hernández Camacho, S., ... Olalde Portugal, V. (2012). Identificación de especies de Fusarium en semilla de ajo en Aguascalientes, México. Revista Mexicana de Micología, 36, 27-32.

Overy, D. P., Valdez, J. G., \& Frisvad, J. C. (2005). Revisions to Penicillium ser. Corymbifera : agents responsible for blue mould storage rot of various flower and vegetable bulbs. Canadian Journal of Botany, 83(11), 1422-1433. https://doi.org/10.1139/b05-110

Patón, L. G., Marrero, M. D. R., \& Llamas, D. P. (2017). In vitro and field efficacy of three fungicides against Fusarium bulb rot of garlic. European Journal of Plant Pathology, 148(2), 321-328.

Pitt, J. I., \& Hocking, A. D. (2009). Fungi and food spoilage (Vol. 519). Springer.

Qin, C. Q., Du, Y. M., \& Xiao, L. (2002). Effect of hydrogen peroxide treatment on the molecular weight and structure of chitosan. Polymer Degradation and Stability, 76(2), 211-218.

Qin, G., Liu, J., Cao, B., Li, B., \& Tian, S. (2011). Hydrogen peroxide acts on sensitive mitochondrial proteins to induce death of a fungal pathogen revealed by proteomic analysis. PLoS One, 6(7), e21945.

Ramos-Guerrero, A., González-Estrada, R. R., Hanako-Rosas, G., Bautista-Baños, S., AcevedoHernández, G., Tiznado-Hernández, M. E., \& Gutiérrez-Martínez, P. (2018). Use of inductors in the control of Colletotrichum gloeosporioides and Rhizopus stolonifer isolated from soursop fruits: in vitro tests. Food Science and Biotechnology, 1-9. https://doi.org/10.1007/s10068-018-0305-5

Reddy, M. V. B., Arul, J., Ait Barka, E., Angers, P., Richard, C., \& Castaigne, F. (1998). Effect of chitosan on growth and toxin production by Alternaria alternata f.sp. lycopersici. Biocontrol Science and Technology, 8(November), 33-43. https://doi.org/10.1080/09583159830414

Singh, D., \& Sharma, R. R. (2018). Postharvest diseases of fruits and vegetables and their management. In Postharvest Disinfection of Fruits and Vegetables (pp. 1-52). Elsevier. 
Recibido:

18/marzo/2019

Aceptado:

11/noviembre/2019
Smilanick, J. L., Mansour, M. F., Gabler, F. M., \& Sorenson, D. (2008). Control of citrus postharvest green mold and sour rot by potassium sorbate combined with heat and fungicides. Postharvest Biology and Technology, 47(2), 226-238. https://doi.org/10.1016/j.postharvbio.2007.06.020

Stanojevic, D., Comic, L., Stefanovic, O., \& Solujic-Sukdolak, S. (2009). Antimicrobial effects of sodium benzoate, sodium nitrite and potassium sorbate and their synergistic action in vitro. Bulg J Agric Sci J, 15(4), 307-311.

Upadhyay, A., Upadhyaya, I., Kollanoor-Johny, A., Baskaran, S. A., Mooyottu, S., Karumathil, D., \& Venkitanarayanan, K. (2013). Inactivation of Listeria monocytogenes on frankfurters by plant-derived antimicrobials alone or in combination with hydrogen peroxide. International Journal of Food Microbiology, 163(2-3), 114-118.

Visagie, C. M., Houbraken, J., Frisvad, J. C., Hong, S.-B., Klaassen, C. H. W., Perrone, G., ... Samson, R. A. (2014). Identification and nomenclature of the genus Penicillium. Studies in Mycology, 78, 343-371.

Wang, L., Wu, H., Qin, G., \& Meng, X. (2014). Chitosan disrupts Penicillium expansum and controls postharvest blue mold of jujube fruit. Food Control, 41, 56-62.

White, T. J., Bruns, T., Lee, S., \& Taylor, J. L. (1990). Amplification and direct sequencing of fungal ribosomal RNA genes for phylogenetics. PCR Protocols: A Guide to Methods and Applications, 18(1), 315-322.

Yahyazadeh, M., Omidbaigi, R., Zare, R., \& Taheri, H. (2008). Effect of some essential oils on mycelial growth of Penicillium digitatum Sacc. World Journal of Microbiology and Biotechnology, 24(8), 1445-1450. https://doi.org/10.1007/s11274-007-9636-8

Yao, H., Tian, S., \& Wang, Y. (2004). Sodium bicarbonate enhances biocontrol efficacy of yeasts on fungal spoilage of pears. International Journal of Food Microbiology, 93(3), 297-304.

Youssef, K., Ligorio, A., Sanzani, S. M., Nigro, F., \& Ippolito, A. (2012). Control of storage diseases of citrus by pre- and postharvest application of salts. Postharvest Biology and Technology, 72(September), 57-63. https://doi.org/10.1016/j.postharvbio.2012.05.004

Youssef, K., \& Roberto, S. R. (2014). Applications of salt solutions before and after harvest affect the quality and incidence of postharvest gray mold of 'Italia' table grapes.

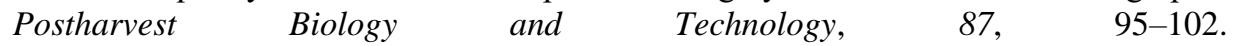
https://doi.org/10.1016/j.postharvbio.2013.08.011 